\title{
Brief Report: Covid Restrictions had Positive and Negative Impacts on Schooling for Students on the Autism Spectrum
}

\author{
Kate Simpson ${ }^{1,2} \oplus \cdot$ Dawn Adams $^{1,2}$ \\ Accepted: 19 January 2022 \\ (c) The Author(s), under exclusive licence to Springer Science+Business Media, LLC, part of Springer Nature 2022
}

\begin{abstract}
The COVID-19 pandemic (2020) resulted in school closures and changes to school delivery. The aim of this study was to explore how these changes impacted on children on the autism spectrum. As part of an online survey, parents $(n=180)$ of school-aged children (9.3-16.5 years) on the autism spectrum in Australia were asked an open-ended question on how the COVID-19 pandemic had impacted on their child's education experience. Nearly half (48\%) of the parents reported only negative impacts, $26 \%$ only positive impacts, $12 \%$ a mix of positive and negative impacts, and $9 \%$ little or no impact. Parents identified that school restrictions impacted on more than their child's learning. These findings highlight areas to consider when supporting autism-friendly learning.
\end{abstract}

Keywords COVID $\cdot$ Learning $\cdot$ Children $\cdot$ Autism $\cdot$ Participation

The COVID-19 pandemic (World Health Organisation, 2020) resulted in stay-at-home orders and shutdowns which posed considerable and unique challenges for families of individuals with neurodevelopmental disorders. There is little research on how children on the autism spectrum and their families cope with life crises or significant disturbances (Latzer et al., 2021) and, prior to COVID-19, there was no research evaluating how individuals on the autism spectrum are affected by pandemics (Mutluer et al., 2020). Pandemics are unique in that they require separation, isolation, and quarantine which result in interference with family and social norms and routines. The DSM-5 diagnostic criteria for autism spectrum disorder includes insistence of sameness and a preference for routine (American Psychiatric Association, 2013). In addition, elevated levels of mental health problems (Lai et al., 2019) and intolerance of uncertainty (Jenkinson et al., 2020) are reported within individuals on the autism spectrum. It would be reasonable to assume that the unpredictable and uncertain events and

Kate Simpson

K.Simpson@Griffith.edu.au

1 Autism Centre of Excellence, School of Education and Professional Studies, Griffith University, Meadowbrook, Logan QLD, Brisbane 4131, Australia

2 Griffith Institute for Educational Research, Griffith University, Brisbane, Australia changes which occurred as a result of the COVID-19 pandemic may bring about specific challenges for children on the autism spectrum.

Studies exploring the experiences of families of children on the autism spectrum during the COVID-19 pandemic suggest that many children showed an increased frequency of behavioural challenges, higher anxiety and poorer emotion regulation, and decreased social communication skills (Nonweiler et al., 2020; Parenteau et al., 2020). By using mixed methods, Oomen et al. (2021) identified not only the burdens (e.g., loss of social contact, increased anxiety and stress) but also the benefits (e.g., reduced sensory and social overload) experienced by adults on the autism spectrum during the pandemic.

One of the most notable impacts of the COVID-19 pandemic restrictions for children was the closure of schools and educational settings. School closures changed both the delivery method for learning and the environment. A systematic review of the COVID-19 research documenting the experiences of individuals on the autism spectrum or their families (Kreysa et al., 2021) identified three articles to February 1, 2021 focused on education across the pandemic. One (Stenhoff et al., 2020) comprises some guidelines for teachers and two report on parental experiences and strategies for home-schooling in Zimbabwe (Majoko \& Dudu, 2020) and the Philippines (Cahapay, 2020). Since this time, another study has explored parental experience of delivering 
home education in Indonesia (Daulay, 2021). A further study (Baten et al., 2021) compared factors predicting students' home learning experiences in children with and without developmental disorders including autism. Baten et al. reported that the teaching methods used during the home learning period were viewed by parents as less effective for children on the autism spectrum compared to their typically developing peers. However, there has been limited research exploring the impact of COVID-19-related changes to the educational experiences of children on the autism spectrum. Children on the autism spectrum are already at an increased risk of poorer educational outcomes, including academic underachievement (Keen et al., 2016). Identifying whether there were any additional challenges or disadvantages experienced or possible benefits due to the COVID-19-related changes is critical and can help inform support going forwards. This is a preliminary study to categorise factors that parents identify as impacting on the educational experiences of children on the autism spectrum due to the COVID-19 pandemic.

\section{Methods}

This study used data collected as part of the Longitudinal Study of Australian Students with Autism (LASA). Ethical approval for this study was obtained from all participating universities and health organisations.

\section{Recruitment and Participants}

The recruitment procedure for the larger longitudinal study is described in Roberts et al. (2018). Briefly, participants were recruited from early intervention centres, autism organisations, parent groups, websites, and mailing lists Australia wide. All children had a parent-reported diagnosis of autism. As described in the protocol paper (Roberts et al., 2018), the parent-reported diagnosis was either verified through a community diagnostic report provided by parents upon entry into the study $(n=129)$, or via their score on the Social Communication Questionnaire (SCQ; Rutter et al., 2008, $n=51$ ).

Parents completed an annual questionnaire. The data for this study were collected during 2020, the year when COVID restrictions were initially enforced. The length and extent of school closures in Australia varied between states. Therefore, to ensure that all parents were providing reflections on the impact after the enforced school closures had finished, all parents were invited to complete the questionnaire after their state government mandated school closures had ended. Parents $(n=180)$ of children $(M$ age $=12.67$ years, $S D=30$ months; $9.3-16.5$ years) participated in this study. Participants were predominantly female $(79.4 \%, n=143)$, in full- or part-time employment $(63.1 \%, n=113)$, and $79.4 \%$ $(n=143)$ reported a gross annual family income equivalent to or above the median household income of Australia of $\$ 80,000$. Children were predominantly male $(79.4 \%)$ and $78.3 \%$ of parents reported at least one co-occurring condition. The most frequently reported co-occurring conditions were anxiety (47.2\%) and attention deficit hyperactivity disorder (36.7\%). Only $3.3 \%$ of children were reported to have an intellectual disability.

\section{Measures and Data Analysis}

Parents were asked to respond to two multiple choice questions to note how their child was schooled before and then during the COVID-19 pandemic. This allowed for the classification of participants into those who continued to attend school, those who transitioned to remote/online learning, and those who were already being home-schooled and continued to be home-schooled during the pandemic. Parents were asked to respond to the following open-ended question: "How has the COVID-19 pandemic impacted on your child's education experience?". Participant responses were analysed using content analysis as used in previous research (e.g., Adams et al., 2019). The first author, who was experienced in content analyses, conducted the analyses. The initial categorisation stage involved reviewing the responses and assigning the data to categories. A process of reviewing, refining, and modifying the categories took place. This was followed by a further categorization process where each category was reviewed to ensure the category encompassed the data, determining if any category was too broad and required splitting into more refined categories, or if categories could be combined under one category. The categories and data were collated in an Excel spreadsheet. Reliability was conducted by a research assistant who was experienced in content analysis. Inter-rater reliability of $20 \%$ of the responses resulted in $82 \%$ agreement (total number of agreed responses/total number of responses coded $\times 100$ ). Items of disagreement were discussed among the researchers and full consensus achieved. The first author then reviewed all categories.

\section{Results}

From the total sample of 180 participants, the majority of parents $(54 \%, n=97)$ identified multiple impacts ranging from two to six different examples. Some parents provided examples that were classified under one category, and other parents provided a number of different categorical responses. Almost half of parents $(48 \%, n=86)$ described COVID restrictions as having a negative impact on their child's education, 26\% $(n=46)$ reported a positive impact, $12 \%(n=22)$ 
reported both positive and negative impacts, and 9\% $(n=16)$ reported little or no impact. Ten (5\%) responses were unclear in terms of impact (e.g., "spent 2 terms doing remote learning") so were not included in the analyses.

Eight categories were generated from the data (see Table 1) and were reported as having either a positive or negative impact on the schooling experience. Parent responses (see Table 1) related to the format of schooling their child received during the pandemic. During Australia's restrictions, the majority of students received remote/online learning $(n=135)$; however, children of essential workers, and vulnerable children who were able to, physically attended school $(n=25)$, and some children continued their independent home-schooling $(n=10)$.

\section{Parent-Reported Impact on Those Who Transitioned to Remote Learning}

Thirty-seven parents (27.4\%) reported positive impacts of remote learning, noting their child "enjoyed", "loved", "thrived" or was more engaged in learning, and grades improved. In contrast, 57 parents (42.2\%) reported a negative impact, with their child doing "no learning" or "bare minimum" and being less engaged and regressing in some learning areas. Four parents commented that their child refused to do schoolwork, as "schoolwork is for school and not home."

Eleven parents $(8.1 \%)$ reported improvements in their child's mental health or well-being including decreased anxiety, aggression, and meltdowns, increased confidence, improved speech, higher participation in extra activities (e.g., cooking, exercise), and bed wetting stopping "almost immediately". Fourteen parents (10.3\%) reported increases in their child's anxiety, self-harming, and depression. Isolation, online classes, coping with learning, and concerns about COVID infection were identified as contributing factors.

Two parents (1.5\%) identified a positive impact on their child's social connections, with some children finding it easier to socialise with smaller numbers and others maintaining social connections online. In contrast, 18 parents (13.3\%) reported negative impacts on social connections, with children missing their friends, feeling isolated, and having less social interaction. Three children (12.0\%) were reported to withdraw socially and to disengage from peers.

Seven children $(5.2 \%)$ benefited from not having to deal with the distraction of other students, movement between classes, and sensory challenges, and having more flexibility made home a positive learning environment for them. In two cases a special study space was created. One child asked for the family caravan to be set up as his classroom where he attended school wearing his uniform. Not all children found the home environment conducive for learning, with $6.6 \%$ of parents reporting distractions from other family members, access to other activities (e.g., playing video games, reading, toys), and a lack of school routine impacting learning.

Sixteen parents $(11.8 \%)$ described the school as "extremely supportive" and providing "incredible home learning". Strategies included daily communication with the teacher (e.g., online meetings, emails), regular facilitated online sessions, and fun learning activities (e.g., virtual camp, visual and performing art activities). Parents also felt supported with "constant communication" and the provision of take-home resources. A lack of these supports was identified as negatively impacting children's learning by $8.8 \%$ of participants, with parents identifying not having access to "any support", having "no online or interactive sessions", requiring "more explicit instruction", having difficulty accessing the curriculum and support staff, and experiencing delays in response to children's questions.

Table 1 Parent reported impacts of COVID-19 on their child's schooling

\begin{tabular}{|c|c|c|c|c|c|c|}
\hline \multirow{3}{*}{$\begin{array}{l}\text { Categories } \\
\text { Little or no impact }\end{array}$} & \multicolumn{2}{|c|}{$\begin{array}{l}\text { Remote/online learning } \\
(n=135)\end{array}$} & \multicolumn{2}{|c|}{$\begin{array}{l}\text { Attended school } \\
(n=25)\end{array}$} & \multicolumn{2}{|c|}{$\begin{array}{l}\text { Home schooled as } \\
\text { usual } \\
(n=10)\end{array}$} \\
\hline & $8(6.0 \%)$ & & $3(12.0 \%)$ & & $5(50.0 \%)$ & \\
\hline & Positive & Negative & Positive & Negative & Positive & Negative \\
\hline Participation and achievement in learning & $37(27.4 \%)$ & $57(42.2 \%)$ & $2(8.0 \%)$ & $3(12.0 \%)$ & $0(0.0 \%)$ & $0(0.0 \%)$ \\
\hline Child mental health and well-being & $11(8.1 \%)$ & $14(10.4 \%)$ & $5(20.0 \%)$ & $9(36.0 \%)$ & $0(0.0 \%)$ & $0(0.0 \%)$ \\
\hline Social activities & $2(1.5 \%)$ & $18(13.3 \%)$ & $2(8.0 \%)$ & $0(0.0 \%)$ & $0(0.0 \%)$ & $0(0.0 \%)$ \\
\hline $\begin{array}{l}\text { Participation in extracurricular school activities (e.g., } \\
\text { library visits, organised physical activities) }\end{array}$ & $0(0.0 \%)$ & $2(1.5 \%)$ & $0(0.0 \%)$ & $0(0.0 \%)$ & $0(0.0 \%)$ & $5(50.0 \%)$ \\
\hline Learning environment, organisation, and structure & $7(5.2 \%)$ & $9(6.7 \%)$ & $3(12.0 \%)$ & $7(28.0 \%)$ & $0(0.0 \%)$ & $0(0.0 \%)$ \\
\hline Support school & $16(11.9 \%)$ & $12(8.8 \%)$ & $6(24.0 \%)$ & $1(4.0 \%)$ & $0(0.0 \%)$ & $0(0.0 \%)$ \\
\hline Support family & $15(11.1 \%)$ & $12(8.8 \%)$ & $0(0.0 \%)$ & $0(0.0 \%)$ & $0(0.0 \%)$ & $0(0.0 \%)$ \\
\hline Whole school transitioning back & $8(5.9 \%)$ & $19(14.1 \%)$ & $0(0.0 \%)$ & $1(4.0 \%)$ & $0(0.0 \%)$ & $0(0.0 \%)$ \\
\hline
\end{tabular}


Remote learning gave a proportion of families $(11.1 \%)$ the opportunity to support their child's learning (e.g., oneto-one assistance). As a result, parents felt they had a better understanding of their child' ability and were able to provide additional targeted support or to fund additional assistance. However, not being able to provide their child with support due to work commitments or having to support multiple children with differing demands was identified as a barrier to learning for $8.8 \%$ of the participants.

Of the 27 parents who commented on the transition back to full-time schooling, 20 (74\%) parents reported a negative experience. They described it as "difficult", "challenging", "a nightmare", their child taking time to settle, the struggle with getting their child to attend, increased absences, and an increase in anxiety, meltdowns, physical aggression, and absconding. Specific factors identified included the rapidity of school return, and either work completed too quickly to catch up or having to wait for other students to catch up on assigned work they had not completed during the remote learning period. The loss of additional support provided, due to reduced staff and staff allocated to different roles, led to an increase in anxiety. Other parents commented that their child settled back well and noted the improvement in their child's learning, independence at drop-off and pick-up, and that their child "realised how much she loves school". The remote learning period provided two parents with more awareness of their child's ability, which resulted in a move to an alternate school support (unit or specialist school).

\section{Parent-Reported Impact on Those Who Continued Attending School}

Both positive and negative impacts on learning were reported, with three parents (12\%) reporting their child "did not learn anything", got lower grades, or had school attendance issues. Two parents (8\%) described it as "successful" with more engagement and lower school refusal. There were also mixed impacts on the child's well-being, with five parents (20\%) commenting that their child coped "amazingly", developed resilience, developed a positive attitude to school, and showed no increase in anxiety. In contrast, seven parents (28\%) reported that their child had difficulty coping, was "unsettled" or "unregulated", and had an increase in aggression, meltdowns, and depression. Only two parents $(8 \%)$ of school attendees noted impacts on social activities; both were positive. One parent noted their child found it easier to play with others and another parent commented that their child found a friend.

Three parents $(12 \%)$ commented that an environment with fewer children, who were socially distanced, suited their child. Six parents (24\%) reported that their child had difficulty with the change in routines, different teachers and classrooms, and social distancing rules.

\section{Impact on Home-Schooled Children}

The only impact noted by parents of home-schooled children $(n=5,50 \%)$ was the impact of reduced participation in community activities that were part of their child's school experience, for example, excursions, library visits, homeschool social groups, and work experience.

\section{Discussion}

The COVID-19 pandemic resulted in changes to both social and educational experiences across the world. In this study, parents of children on the autism spectrum in Australia identified both positive and negative impacts of the changes to educational provision for their children during the COVID-19 school closures. Across the entire sample, almost half (48\%) of parents reported only negative impacts, just over a quarter (26\%) only positive impacts, and a further $12 \%$ reported a mix of positive and negative impacts. The identification of both positive and negative impacts of COVID-19 restrictions aligns with a large $(n=1044)$, multicountry mixed-methods study of adults on the autism spectrum (Oomen et al., 2021) and highlights the importance of using methods such as open-ended responses to collate information on a broad range of experiences during such an unprecedented time.

Parents identified different areas of support and barriers to their children's educational experiences during the COVID-19 school closures. There was variability in frequency of the reported categories across those who had and had not experienced a change to their educational delivery method during the pandemic. The only impact noted by parents of children who were home-schooled both before and during COVID-19 restrictions was a reduction in the number of extracurricular activities that could be undertaken due to shutdowns. This may have been particularly notable for this group of children given that home-educated children are reported to be more involved in extracurricular and social activities than those in mainstream education (for a review on home-schooling in autism see O'Hagan et al., 2021).

Children of essential workers and vulnerable children were able to continue physically attending during school closures; this represented $13.8 \%$ of the sample reported upon in this paper. Despite attending the same physical location to receive their education, these children were still reported to be impacted by the changes. The smaller numbers of students attending school resulted in $24 \%$ of parents reporting their child received more support from the school. However, 
$28 \%$ of parents reported that the changes in class organisation and teachers and disruption to the school routine negatively impacted on their child. This is consistent with adults on the autism spectrum who found the loss of routine one of the challenges related to the pandemic (Oomen et al., 2021).

Parents reported that the changes due to the pandemic had a positive and negative impact on the child's mental health and well-being. Both positive and negative impacts were reported in children who moved to remote/online learning $(8.1 \%, 10.4 \%$ respectively) and children who continued to physically attend school during the pandemic restrictions (20\%,36.0\% respectively). This highlights how complex the factors impacting mental health and well-being may be for children on the autism spectrum. It also aligns with Vasa et al.'s (2021) finding that $59 \%$ of children on the autism spectrum experienced worsening or new psychiatric problems during the COVID-19 pandemic and $41 \%$ did not. Risk factors for increased psychiatric problems reported by Vasa et al. included having an individual with COVID-19 diagnosed in the family, child understanding of COVID-19, and parent psychopathology. Children's concern about COVID19 was also reported by parents in the current study and by Baten et al. (2021) as a factor impacting on their child's mental health. However, Vasa et al. did not measure other potential contributing factors such as changes to routines, support accessed, or changes to social and educational provisions. Given these were highlighted by parents in this study it would be interesting to explore how they may interact with the significant predictors identified by Vasa et al., such as parental psychopathology.

Educational experiences during the COVID-19 school closures were also mixed for children who had previously been at school but then had to stay at home and access their learning through online platforms. A little over a quarter of the children (27.4\%) were reported to experience a positive impact academically and $8.1 \%$ showed improvements in their mental health and well-being. However, for $42.2 \%$ of children, online learning resulted in reduced participation and lower achievement. This is somewhat unsurprising given that children were required to adapt to new ways of learning. Not only were they expected to learn whilst within an environment they typically associated with free time, but they were also required to attend to online instructional content, to apply a range of traditional and digital literacy skills, and to remain engaged while often having to inhibit any desires to use the same computer or tablet for their normal activities (e.g., playing games, watching videos; Stenhoff et al., 2020). Challenges due to teaching methods were also reported by Baten et al. (2021) who identified that teaching strategies used in online were less effective for children on the autism spectrum. It is important that those teaching and supporting students on the autism spectrum recognise the potential negative impact on learning across this period and provide structured supports and opportunities to recover this time. This needs to include missed opportunities to learn the "hidden curriculum" (i.e., implicit social rules and social norms in the school environment), which is a prerequisite for success in the school years and beyond (Reicher, 2020).

A number of articles exploring the impact of the pandemic have identified the importance of using it as an opportunity to shift to alternative ways of supporting people, communicating and accessing social spaces that are accessible and supportive for all (see section "Discussion" in Cassidy et al., 2020). Parents in this study highlighted how the changes to the physical and/or social environment of school can impact either positively or negatively on their child's participation, achievement, and well-being. The COVID-19 experiences of children and adolescents on the autism spectrum have provided a "natural experiment" of the impact of changed environments and have shown that when children on the autism spectrum are able to develop and interact in an environment which balances demands and challenges with well-being, the children are more able to participate, communicate, and interact with others (Mumbardó-Adam et al., 2021). Such findings on what helped and what hindered outcomes during this pandemic may indeed go towards "(making) the world a more autism friendly place" (Spain et al., 2021, 1).

\section{Limitations and Future Directions}

This study should be considered in the context of its limitations. These data were taken from parents who had already committed 6 years to a longitudinal study, which may introduce a sampling or selection bias. The lack of independent assessment to confirm autism diagnosis, and the reliance on a screener of autism characteristics to support the parent-reported diagnosis in 51 participants, are additional limitations.

All the families were from a single country, so views and perspectives may not be representative of countries where lockdowns were longer, more severe, or less effective. The limitation of coding data from a single question is acknowledged, although the depth of the answers provided by parents highlights the complexity of supporting children on the autism spectrum and identifies key categories that can be both supports and barriers to the child's learning environment. The small number of responses in some categories suggests the salience of factors varied for parents and further investigation is required to determine if these are generalisable to the broader population of autistic and non-autistic students; follow-up studies should be undertaken to explore the extent to which these are long-term effects of the pandemic.

As schools across the world are expected to face ongoing impacts of COVID on educational delivery, it is important for educational departments and schools to be aware 
of the wide-ranging impact of school closures for students on the autism spectrum. The breadth of these impacts may not only be measurable in academic output but may need to be purposefully monitored through measures of student well-being or mental health. It is also important to acknowledge the impact on those who continue to attend school but without many of their peers or teachers; such changes to the routine and environment can have a notable influence on mental health and well-being, changes which may have been attributed to the broader challenges of the pandemic. Finally, the findings from this study identify preliminary areas for future attention in a larger group of participants to ensure that students' learning experiences continue to be supported throughout times of change and crises, such as that experienced in the previous 2 years.

Acknowledgments The authors are grateful to the children with autism and their families for giving their time to support this research study. The authors acknowledge the work of the LASA team members: Jacqui Roberts (Project Leader), Susan Bruck, Trevor Clark, Sandra Devaraj, Robyn Garland, Honey Heussler, Antoinette Hodge, Patricia Howlin, Deb Keen, Jessica Paynter, Natalie Silove, David Trembath, Madonna Tucker, Marleen Westerveld and Katrina Williams.

Author Contributions KS and DA contributed to the conception of the study, design and coordination and manuscript writing. KS conducted the content analysis and interpretation of the data. All authors read and approved the final manuscript.

Funding The authors acknowledge the financial support of the Cooperative Research Centre for Living with Autism (Autism CRC), established and supported under the Australian Government's Cooperative Research Centres Program. The views expressed are those of the author(s) and not necessarily those of any of the funding bodies.

\section{Declarations}

Conflict of interest The authors report no conflict of interest.

\section{References}

Adams, D., Young, K., Simpson, K., \& Keen, D. (2019). Parent descriptions of the presentation and management of anxiousness in children on the autism spectrum. Autism, 23, 980-992. https:// doi.org/10.1177/1362361318794031

American Psychiatric Association. (2013). Diagnostic and statistical manual (5th ed.). American Psychiatric Publishing.

Baten, E., Vlaeminck, F., Mues, M., Valcke, M., Desoete, A., \& Warreyn, P. (2021). The impact of school strategies and the home environment on home learning experiences during the COVID-19 pandemic in children with and without developmental disorders. Journal of Autism and Developmental Disorders. https://doi.org/ 10.1007/s10803-021-05383-0

Cahapay, M. B. (2020). How Filipino parents home educate their children with autism during COVID-19 period. International Journal of Developmental Disabilities. https://doi.org/10.1080/20473869. 2020.1780554

Cassidy, S. A., Nicolaidis, C., Davies, B., Rosa, S. D. R., Eisenman, D., Onaiwu, M. G., Kapp, S. K., Kripke, C. C., Rodgers,
J., \& Waisman, T. (2020). An expert discussion on autism in the COVID-19 pandemic. Autism in Adulthood, 2(2), 106-117. https://doi.org/10.1089/aut.2020.29013.sjc

Daulay, N. (2021). Home education for children with autism spectrum disorder during the COVID-19 pandemic: Indonesian mothers experience. Research in Developmental Disabilities, 114, 103954. https://doi.org/10.1016/j.ridd.2021.103954

Jenkinson, R., Milne, E., \& Thompson, A. (2020). The relationship between intolerance of uncertainty and anxiety in autism: A systematic literature review and meta-analysis. Autism, 24(8), 19331944. https://doi.org/10.1177/1362361320932437

Keen, D., Webster, A., \& Ridley, G. (2016). How well are children with autism spectrum disorder doing academically at school? An overview of the literature. Autism, 20, 276-294. https://doi.org/ $10.1177 / 1362361315580962$

Kreysa, H., Schneider, D., Kowallik, A. E., Dastgheib, S. S., Dogdu, C., Kühn, G., Ruttloff, J. M., \& Schweinberger, S. R. (2021). Psychosocial and behavioural effects of the COVID-19 pandemic on children with autism and their families: A systematic review and initial data from a multinational online survey. https://doi.org/10. 31234/osf.io/7dwce

Lai, M.-C., Kassee, C., Besney, R., Bonato, S., Hull, L., Mandy, W., Szatmari, P., \& Ameis, S. H. (2019). Prevalence of co-occurring mental health diagnoses in the autism population: A systematic review and meta-analysis. The Lancet Psychiatry, 6(10), 819-829. https://doi.org/10.1016/S2215-0366(19)30289-5

Latzer, I. T., Leitner, Y., \& Karnieli-Miller, O. (2021). Core experiences of parents of children with autism during the COVID-19 pandemic lockdown. Autism, 25(4), 1047-1059. https://doi.org/ $10.1177 / 1362361320984317$

Majoko, T., \& Dudu, A. (2020). Parents' strategies for home educating their children with autism spectrum disorder during the COVID19 period in Zimbabwe. International Journal of Developmental Disabilities. https://doi.org/10.1080/20473869.2020.1803025

Mumbardó-Adam, C., Barnet-López, S., \& Balboni, G. (2021). How have youth with autism spectrum disorder managed quarantine derived from COVID-19 pandemic? An approach to families perspectives. Research in Developmental Disabilities, 110, 103860. https://doi.org/10.1016/j.ridd.2021.103860

Mutluer, T., Doenyas, C., \& Genc, H. A. (2020). Behavioral implications of the Covid-19 process for autism spectrum disorder, and individuals' comprehension of and reactions to the pandemic conditions. Frontiers in Psychiatry. https://doi.org/10.3389/fpsyt. 2020.561882

Nonweiler, J., Rattray, F., Baulcomb, J., Happé, F., \& Absoud, M. (2020). Prevalence and associated factors of emotional and behavioural difficulties during COVID-19 pandemic in children with neurodevelopmental disorders. Children, 7(9), 128. https://www. mdpi.com/2227-9067/7/9/128

O'Hagan, S., Bond, C., \& Hebron, J. (2021). What do we know about home education and autism? A thematic synthesis review. Research in Autism Spectrum Disorders, 80, 101711. https://doi. org/10.1016/j.rasd.2020.101711

Oomen, D., Nijhof, A. D., \& Wiersema, J. R. (2021). The psychological impact of the COVID-19 pandemic on adults with autism: A survey study across three countries. Molecular Autism, 12(1), 21. https://doi.org/10.1186/s13229-021-00424-y

Parenteau, C. I., Bent, S., Hendren, R. L., Hossain, B., Chen, Y., Widjaja, F., \& Breard, M. (2020). A qualitative analysis: The experience of parents of children with autism spectrum disorder during the COVID-19 pandemic. Journal of the American Academy of Child and Adolescent Psychiatry, 59(10), S251. https://doi.org/ 10.1016/j.jaac.2020.08.411

Reicher, D. (2020). Debate: Remote learning during COVID-19 for children with high functioning autism spectrum disorder. Child 
and Adolescent Mental Health, 25(4), 263-264. https://doi.org/ 10.1111/camh. 12425

Roberts, J., Adams, D., Heussler, H., Keen, D., Paynter, J., Trembath, D., Westerveld, M., \& Williams, K. (2018). Protocol for a prospective longitudinal study investigating the participation and educational trajectories of Australian students with autism. BMJ Open, 8(1), e017082. https://doi.org/10.1136/bmj-open-2017-017082

Rutter, M., Bailey, A., \& Lord, C. (2008). The social communication questionnaire. Western Psychological Services.

Spain, D., Mason, D., Capp, S. J., Stoppelbein, L., White, S. W., \& Happé, F. (2021). "This may be a really good opportunity to make the world a more autism friendly place": Professionals' perspectives on the effects of COVID-19 on autistic individuals. Research in Autism Spectrum Disorders, 83, 101747. https://doi.org/10. 1016/j.rasd.2021.101747

Stenhoff, D. M., Pennington, R. C., \& Tapp, M. C. (2020). Distance education support for students with autism spectrum disorder and complex needs during Covid-19 and school closures. Rural Special Education Quarterly, 39(4), 211-219. https://doi.org/10. $1177 / 8756870520959658$

Vasa, R. A., Singh, V., Holingue, C., Kalb, L. G., Jang, Y., \& Keefer, A. (2021). Psychiatric problems during the COVID-19 pandemic in children with autism spectrum disorder. Autism Research. https:// doi.org/10.1002/aur.2574

World Health Organisation. (2020). Coronavirus disease (COVID-19): Situation report-202. https://www.who.int/docs/default-source/ coronaviruse/situation-reports/20200809-covid19-sitrep-202. pdf?sfvrsn=2c7459f6_2

Publisher's Note Springer Nature remains neutral with regard to jurisdictional claims in published maps and institutional affiliations. 